\title{
TEMA 3. LAS COMPETENCIAS DE LA UNIÓN EUROPEA
}

Catedrático Dr. Valentín Bou Franch Curso académico 2016-2017

VNIVERSITAT Ë̈̈VALẼNCIA

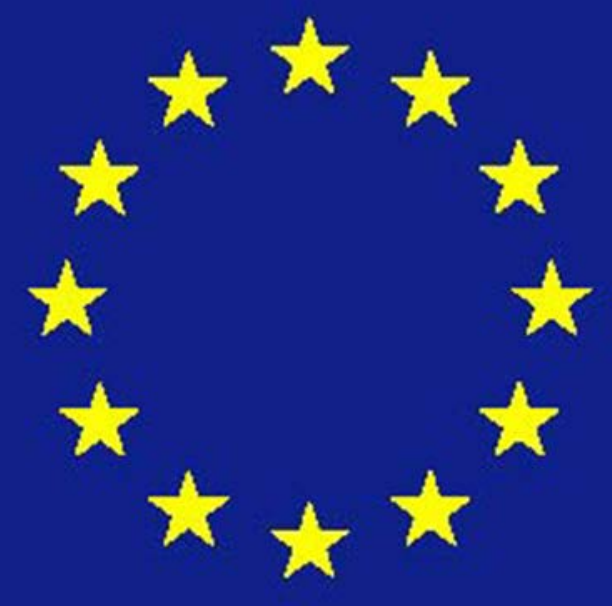




\section{LA ATRIBUCIÓN DE COMPETENCIAS A LA UNIÓN EUROPEA}

A. PRECISIONES CONCEPTUALES

B. EL PRINCIPIO DE ATRIBUCIÓN DE COMPETENCIAS Y LA UE

C. COMPETENCIAS EXPLÍCITAS E IMPLÍCITAS

D. LA CLÁUSULA DE IMPREVISIÓN: LA EXPLICITACIÓN DE LAS COMPETENCIAS IMPLÍCITAS

E. LAS COMPETENCIAS RESERVADAS A LOS ESTADOS MIEMBROS 


\section{A. PRECISIONES CONCEPTALES}

1. $\neq$ entre competencias de los Estados y de las Organizaciones Internacionales (=UE)

○ Estados $=$ entes de base territorial $\rightarrow$ compts. soberanas (todas las compts.)

○ Las OI = entes de finalidad funcional $\rightarrow$ competencias de atribución:

- Sin atribución $\rightarrow$ Ol incompetente $\rightarrow$ acto ultra vires

- Compts. de la UE: son compts. específicas, concretadas caso por caso en los tdos. constitutivos

○ No delimitación competencial por materias

- Competencias de OI (=UE) son competencias funcionales

O Importancia objetivos o fines de cada OI Art. 1, §1 TUE, tx. 6, p. 50 
1) $U E=$ OI intergubernamental $\rightarrow$ tendrá competencias de atribución

2) Principio de atribución de competencias explicitado en el TUE y TFUE

○ Art. 1, §1 TUE, p. 50

$\odot$ Art. 3.6 TUE, p. 51

$\odot$ Art. 5.1 TUE, p. 52

$\odot$ Art. 7 TFUE, p. 78.

3) Atribución de competencias a la UE

○ Debe existir un artículo en los Tdos. que así lo prevea

○ Doble límite:

- Art. 5.2, lín. 1 TUE, p. 52 límite a la actuación de la UE

- Art. 13.2 TUE, p. 55 límite a la actuación de las instituciones europeas

$\odot$ Si se excede cualquier límite $\rightarrow$ acto ultra vires $\rightarrow$ recurso de nulidad

- Garantía de la correcta aplicación de los Tdos.

- Control judicial de la competencia de la UE

$\searrow$ Obligación de motivar (requisito de validez) Art. 296, §2 TFUE, p. 168. Abarca:

- Indicar la(s) base(s) jurídica(s) de cada acto de la UE

- Indicar las fases del procedimiento de adopción

o Indicar los motivos o razones de su adopción 
4) Las competencias atribuidas a la UE ¿son irreversibles? ¿pueden ser recuperadas por los EEMM?

○ Jurisprudencia TJCE: son irreversibles, salvo revisión de los Tdos.

- Tx. 70, St. Costa c. ENEL, p. 413, §7

- Tx. 29, St. Agencia de Abastecimiento, p. 272, §18-20

○ Reinterpretar por Tdo. Lisboa:

- Art. 48.2 TUE, p. 71: revisar los Tdos. para aumentar o REDUCIR las compts. atribuidas a la UE

o Declaración 18 del Acta final Tdo. Lisboa lo repite

- La UE puede decidir dejar de ejercer compts. compartidas con los EEMM Art. 2.2 in fine TFUE, p. 76

- Se prevé la posible retirada de un Estado miembro Art. 50 TUE, pp. 72-73

o Discurso David Cameron 22-1-2013

\section{COMPETENCIAS EXPLÍCITAS E IMPLÍCITAS}

1) El p. de atribución de compts. ¿significa que la UE sólo tiene las compts. explícitas o expresamente atribuidas en los Tdos.?

○ No, por doctrina de las competencias implícitas o tácitas

2) Reconocimiento jurisprudencial de las compts. implícitas de la UE

○ Tx. 26, St. Fédéchar 1956, p. 261

○ Tx. 27, St. Italia c. Alta Autoridad 1960, pp. 263-264

○ Tx. 28, St. AETR 1971, pp. 267 y ss., §12-14, 17-19, 22, 28 y 31 
D. LA CLÁUSULA DE IMPREVISIÓN: LA EXPLICITACIÓN DE LAS COMPETENCIAS IMPLÍCITAS

1) Originalidad de la UE: desde inicios CCEE, previsión de un procedimiento para explicitar las competencias implícitas Art. 352.1 TFUE, p. 183

2) Novedades Tdo. Lisboa:

○ Requiere aprobación previa del PE Art. 352.1 TFUE, p. 183

○ Por principio de subsidiariedad, sometido a control de Parlamentos nacionales Art. 352.2 TFUE, p. 183

\section{LAS CONDICIONES PARA APLICAR LA CLÁUSULA DE IMPREVISIÓN}

1) Cuatro condiciones para aplicar cláusula de imprevisión (art. 352 TFUE):

- Sólo para lograr un objetivo de la UE

- Que la acción de la UE sea necesaria para alcanzar uno de sus objetivos (no en la PESC)

- PERO Tx. 28, §95, p. 271

○ Sin competencias explícitas previstas en los Tdos. constitutivos

- TJUE afirma el carácter subsidiario del art. 352 TFUE

๑ Supuestos en los que está totalmente prohibido utilizar el art. 352 TFUE:

- (tradicional) exclusión de la PESC Art. 352.4 TFUE, p. 184

- (novedad Tdo. Lisboa) Art. 352.2 TFUE, p. 183

LOS LÍMITES A LA UTILIZACIÓN DE LA CLÁUSULA DE IMPREVISIÓN

1) Reacción del TJCE al uso abusivo de esta cláusula: no puede ser un medio de aumentar las compts. de las CCEE, sin revisar previamente los Tdos.

○ Dictamen 1996 CEDH, §29-30 


\section{E. LAS COMPETENCIAS RESERVADAS A LOS ESTADOS MIEMBROS}

1) Las competencias no atribuidas (explícita o implícitamente) a la UE siguen perteneciendo a los EEMM

2) Explicitado reiteradamente en el TUE

○ Art. 4.1 TUE, p. 51

$\odot$ Art. 5.2 TUE, p. 52

3) La función de los Tdos. constitutivos no es enumerar las compts. reservadas a los EEMM

- PERO En ocasiones, se limitan las compts. de la UE, especificando las compts. reservadas a los EEMM, p. ej. Art. 153.5 TFUE, p. 124

4) EI DUE puede condicionar, de manera limitada, el ejercicio discrecional de compts. soberanas reservadas a los EEMM (p. ej. Interpretación del TJCE de "orden público", "seguridad pública", etc.) 


\section{LA DELIMITACIÓN DE LAS COMPETENCIAS DE LA UNIÓN EUROPEA}

A. INTRODUCCIÓN

B. LAS COMPETENCIAS EXCLUSIVAS

C. LAS COMPETENCIAS COMPARTIDAS

D. LAS COMPETENCIAS DE APOYO, COORDINACIÓN Y COMPLEMENTO

E. LA COMPETENCIA ESPECIAL EN MATERIA DE POLÍTICA EXTERIOR Y DE SEGURIDAD COMÚN (PESC) 


\section{A. INTRODUCCIÓN}

1) Novedad Tdo. Lisboa: delimita por primera vez las competencias de la UE ( $\leftarrow$ por presión Länders alemanes)

○ Sigue doctrina y jurisprudencia previas

○ Para ello:

- distingue diversas categorías de competencias Art. 2 TFUE, p. 78

- clasifica los "ámbitos" (no materias) de cada categoría de competencias Arts. 3-6 TFUE, pp. 76-78

\section{B. LAS COMPETENCIAS EXCLUSIVAS}

1) Concepto: Art. 2.1 TFUE, p. 76

○ Consecuencia: excluye acción normativa EEMM Tx. 30, p. 275 §7 a p. 276 §

○ PERO la exclusión de los EEMM no es absoluta

- Previsión del Art. 2.1 in fine TFUE, p. 76

- Si no hay ejercicio efectivo de la compt. exclusiva por la UE, compt. transitoria de los EEMM Tx. 31, pp. 280-281, §30, 33-34 y 39-40

2) Lista cerrada de ámbitos en los que la UE tiene compt. exclusiva Art. 3 TFUE, pp. 76-77 
1) Concepto residual: Art. 4.1 TFUE, p. 77

2) Consecuencias Art. 2.2 TFUE, p. 76

$\odot$ ¿Competencias concurrentes? No

๑ Primacía acción UE

3) No asimilar compts. compartidas ejercidas por la UE con compts. exclusivas de la UE:

○ La UE sólo puede ejercer una compt. compartida si cumple el p. de subsidiariedad

- (Protocolo 25) el acto adoptado por la UE al ejercer una compt. compartida no agota el ámbito en el que la UE tenga este tipo de compt.

○ Si la UE decide dejar de ejercer una compt. compartida, los EEMM la recuperan

4) Pese a la definición residual, el TFUE identifica los ámbitos "principales" Art. 4.2 TFUE, p. 77

5) Emergen compts. compartidas "especiales" (la acción de la UE no excluye la de los EEMM):

- Políticas económicas (Arts. 2.3 y 5.1 TFUE)

○ Políticas de empleo (Arts. 2.3 y 5.2 TFUE)

○ La investigación, el desarrollo tecnológico y el espacio (Art. 4.3 TFUE)

- La cooperación para el desarrollo y la ayuda humanitaria (Art. 4.4 TFUE)

○ Las políticas sociales (Art. 5.3 TFUE)

๑ Por remisión del Art. 2.6 TFUE, otras que aparezcan en el TFUE (p. ej. Art. 114.4 y 5) 
1) Acción UE complementa acción EEMM

2) Concepto y límite Art. 2.5 TFUE, p. 76

3) Ámbitos "cerrados" de este tipo de compt. Art. 6 TFUE, pp. 77-78

E. LA COMPETENCIA ESPECIAL EN MATERIA DE POLITICA EXTERIOR Y DE SEGURIDAD COMÚN

1) Por su importancia, no se reconduce a las tres clasificaciones anteriores

2) La compt. especial es la Política Exterior y de Seguridad Común (PESC), incluida una Política Común de Seguridad y Defensa (PCSD) Art. 2.4 TFUE, p. 76

3) Principales razones de la "especialidad" de la PESC:

○ Art. 24.1, §2 TUE, pp. 61-62

$\odot$ Art. 42.2 TUE, p. 68, etc. 


\section{LOS PRINCIPIOS QUE RIGEN EL EJERCICIO DE LAS COMPETENCIAS}

A. EL PRINCIPIO DE SUBSIDIARIEDAD

A.1. El alcance del principio de subsidiariedad

A.2. Los controles de la aplicación del principio de subsidiariedad

B. EL PRINCIPIO DE PROPORCIONALIDAD 
1) Regulado en:

\section{A.1. EL ALCANCE DEL PRINCIPIO DE SUBSIDIARIEDAD}

○ el TUE:

- Preámbulo, párrafo antepenúltimo TUE, p. 50

- Art. 5.3 TUE, p. 52

- Protocolo no 2 sobre la aplicación de los principios de subsidiariedad y proporcionalidad

2) Competencias a las que afecta: competencias no exclusivas de la UE. Son:

○ Las compts. compartidas

○ Las compts. de apoyo, coordinación y complemento

3) El p. de subsidiariedad limita el ejercicio de las competencias de la UE a las que se aplica:

- Este p. determina si la UE debe intervenir o no, ejerciendo su competencia (NO se presume)

○ Si debe intervenir, será sólo en la medida en que esté justificado

4) La intervención UE debe justificarse (Protocolo no 2, antes, jurisprudencia TJCE Tx. 35, §26-

27, pp. 313-314):

- ante la insuficiencia de la acción de los EEMM (a nivel central, regional y local)

○ por una mejor eficacia de la acción común

$\odot$ por las dimensiones supranacionales de la acción

5) Doble perspectiva del principio de subsidiariedad:

○ Defiende las compts. de los EEMM: la UE sólo intervendrá cuando sea estrictamente necesario

○ Afirma la nec. de la intervención de la UE, cuando esté justificado 


\section{A.2. LOS CONTROLES DE LA APLICACIÓN DEL PRINCIPIO DE SUBSIDIARIEDAD}

1) Regulado en: Arts. 5.3, $\$ 2$ y 12.b) TUE; en Protocolo no 1 sobre el cometido de los Parlamentos nacionales en la UE; y en Protocolo no 2 sobre la aplicación de los ps. de subsidiariedad y proporcionalidad

2) El control previo por los Parlamentos nacionales

○ Es control político (preventivo, a priori) (=“sistema de alerta temprana")

$\odot$ Consiste en:

- Oblig. de remitir a Parlamentos nacionales ( $\rightarrow$ "regionales") información sobre todo proyecto de acto legislativo

- Cada Parlamento nacional emite un dictamen motivado en 8 semanas si considera que el proyecto de acto legislativo es contrario al p. de subsidiariedad

o Dos dictámenes si es Parlamento bicameral

- Si 1/3 Parlamentos nacionales emiten voto negativo: la Comisión debe re-examinar su propuesta

- Si mayoría votos negativos, el PE o el Consejo podrán devolver automáticamente a la Comisión su propuesta

3) El control posterior del Tribunal de Justicia

$\odot$ Es control judicial (a posteriori)

○ Oblig. de motivar los actos legislativos en relación con los ps. de subsidiariedad y de proporcionalidad

$\odot$ Recurso de nulidad presentado por EM "de conformidad con su Derecho interno" en nombre de su Parlamento nacional 
1) Regulado en:

$\odot$ Art. 5.4 TUE, p. 52

○ Protocolo no 2

2) Competencias a las que afecta: a todas las competencias de la UE

3) Diferencias entre ps. de:

- Subsidiariedad: determina si la UE debe o no intervenir, ejerciendo su competencia

๑ Proporcionalidad: determina el alcance de la intervención requerida de la UE

4) Persigue evitar los excesos legislativos de la UE

○ Jurisprudencia TJ Tx. 35, p. 315, §54

○ Art. 5.4 TUE y Protocolo no 2: el p. de proporcionalidad se aplica:

- Al contenido de los actos de la UE: las cargas económicas y administrativas serán lo más reducidas posible y proporcionales al objetivo a alcanzar

- A la forma de los actos de la UE: tradicionalmente, se prefieren las directivas a los reglamentos europeos (dejan + margen de apreciación a los EEMM) 


\section{EL EJERCICIO DE LAS \\ COMPETENCIAS DE LA UNIÓN POR "ALGUNOS" EEMM}

A. INTRODUCCIÓN

B. LAS FÓRMULAS DE LA EXCLUSIÓN VOLUNTARIA

C. LAS COOPERACIONES REFORZADAS

C.1. Los antecedentes previos

C.2. El régimen de las cooperaciones reforzadas

D. MECANISMOS DE FLEXIBILIDAD ADICIONALES EN EL ÁMBITO DE LA POLÍTICA EXTERIOR Y DE SEGURIDAD COMÚN 
1) Todo tdo. internacional (incluidos los Tdos. constitutivos UE) se debaten entre:

○ P. de la integridad del texto del tratado (=igualdad de derechos y deberes para todos)

$\odot$ P. de la flexibilidad del texto del tratado (=regímenes diferenciados especiales para algunos Es.)

2) En el proceso de integración europea:

○ Antes del Tdo. de Maastricht, los Tdos. constitutivos de las CCEE siguieron, en general, el p. de la integridad del texto del tdo.

- Excepción: los "períodos transitorios" de las Actas de adhesión de nuevos EEMM (=p. flexibilidad)

- Esta posibilidad todavía persiste

○ Con Tdo. Maastricht aparece objetivo político + ambicioso: la creación de la UE

- Evidencia que:

- No todos los EEMM tienen el mismo concepto de una Europa Unida

o No todos EEMM quieren avanzar en profundización integración europea a la misma velocidad

$\varnothing$ Por unanimidad en reforma Tdos., ir al ritmo de los más lentos salvo que aparezcan fórmulas nuevas de flexibilización

3) Con Tdo. Maastricht, aparecen las "fórmulas de exclusión voluntaria" (dimensión negativa)

- Uno o varios EEMM deciden voluntariamente (y consensuado con resto EEMM) autoexcluirse (opting out) del ejercicio de algunas compts. atribuidas a CCEE ó UE

4) Con Tdo. Ámsterdam, aparecen "cooperaciones reforzadas" (dimensión positiva)

○ Algunos EEMM irán en la vanguardia de la integración europea, permitiendo que el resto de EEMM se sume posteriormente 
1) En la creación de la moneda única ("euro") desde Tdo. Maastricht

- Protocolos sobre la situación especial de Reino Unido y Dinamarca

- Se excluyen voluntariamente del establecimiento del euro (=opting out)

- Posteriormente podrán incorporarse (=opting in) si cumplen las condiciones

๑ El euro no es acervo comunitario $\rightarrow$ los Es. que se adhieran después, podrán elegir

- Suecia se autoexcluyó al adherirse como nuevo EEMM (opting out + opting in)

2) En la Política Social, con Tdo. Maastricht

○ con Tdo. Maastricht, por Margaret Tacher, la Política Social no en TCE, sino en un Protocolo especial que excluye al Reino Unido (opting out)

○ Con Tdo. Ámsterdam, con Tony Blair, se deroga el Protocolo especial y la Política Social se incluye en el TCE para todos los EEMM (opting in del Reino Unido)

3) En el Espacio de Libertad, Seguridad y Justicia (ELSJ)

○ Situación especial de Reino Unido e Irlanda

- Con Tdo. Maastricht, Reino Unido acepta compt. de la UE sobre asilo e inmigración (tercer pilar: la CAJAI)

- Tdo. Ámsterdam "comunitariza" estas compts (son una parte del ELSJ). Al incluirlas en el TCE $\rightarrow$ RU se opone a renunciar compt. sobre control fronteras exteriores $\rightarrow$ Afecta a Irlanda, por tdo. int'l. previo con RU $\rightarrow$ Fórmulas de exclusión voluntaria

○ RU (=opting out absoluto): podrá establecer control fronteras sobre resto EEMM

○ RU + Irlanda (opting out relativo) entre sí: pueden celebrar los tdos. int'les que deseen sobre el control de sus fronteras

o Resto de EEMM respecto de RU (opting out recíproco) pueden establecer controles en fronteras exteriores que deseen 
- Tdo. Lisboa: "comunitariza” todo el ELSJ

o Se extiende a todo el ELSJ el derecho de opting out con posibilidad de opting in de RU + Irlanda

๑ Situación especial de Dinamarca

- Dinamarca también se opuso a la "comunitarización" de la CAJAI $\rightarrow$ fórmulas flexibles:

- Derecho general de opting out respecto de cualquier medida adoptada en el ELSJ

o En seis meses desde adopción, derecho de opting in respecto de una medida concreta

$\varnothing$ En este caso, celebrar un Tdo. int'l UE-Dinamarca (no por normas Do. derivado europeo)

- En cualquier momento podrá renunciar a toda su situación especial

4) Carta de los Derechos Fundamentales de la Unión Europea (con Tdo. Lisboa)

○ Polonia y Reino Unido (¿Chequia? NO) se excluyen voluntariamente (opting out) de su aplicación

○ No hay prevista ninguna formula de opting in

\section{LAS COOPERACIONES REFORZADAS}

1) El acervo de Schengen

\section{C.1. LOS ANTECEDENTES PREVIOS}

○ Los acuerdos Schengen de 1985 y 1990 y su acervo se negociaron al margen de los Tdos. constitutivos

- Reino Unido e Irlanda se negaron a participar en el espacio Schengen (autoexcluidos)

- Sí se añadieron Estados terceros a las CCEE (Islandia y Noruega) 
○ Tdo. de Ámsterdam:

- Estableció integración acervo Schengen en el marco de la UE

- Art. 1 Protocolo no 2: es una "cooperación reforzada" que afecta a 13 de los 15 EEMM (excluidos Reino Unido e Irlanda, por no obligarse por acuerdos Schengen)

o Protocolo no 4: pueden solicitar en cualquier momento participar en algunas o en todas las disposiciones de dicho acervo

- Posición "especial" de Dinamarca (Protocolo no 5): vinculada por el acervo Schengen, aunque no por todas las consecuencias de su comunitarización. Si decidiera vincularse $\rightarrow$ tdo. CE-Dinamarca

○ Tdo. de Lisboa: la situación se mantiene, ampliada a todo el ELSJ

- Vincula a 26 de los 28 EEMM (="cooperación reforzada")

- Reino Unido e Irlanda excluidos

$\circ$ Protocolo no 20: mantienen control de sus fronteras

o Protocolo no 21: opting out del Título V TFUE, complementado con opting in en cualquier momento

- Posición "especial" Dinamarca (Protocolo no 22): opting out general, pero con derecho de opting in internacional en seis meses siguientes a la adopción de medidas

- Peculiaridades del "acervo Schengen (="cooperación reforzada") respecto del régimen gnral. de las cooperaciones reforzadas:

- el Acervo Schengen se considera acervo de la UE $\rightarrow$ debe ser aceptado en su totalidad por los nuevos EEMM

- Es una cooperación reforzada en la que pueden participar Es. terceros a la UE:

o Desde el inicio, Islandia y Noruega

○ Abierta a otros Estados: Suiza y Liechtenstein 
2) La implantación del “euro" como moneda única

○ Reino Unido, Dinamarca y Suecia se autoexcluyeron del euro

○ De los 25 EEMM restantes, sólo 17 (inicialmente, 11) están en la moneda única

○ Los 8 EEMM restantes, obligación de cumplir condiciones para ingresar en el Eurogrupo

○ El euro es una auténtica cooperación reforzada Arts. 136 y 137 TFUE, pp. 117-118

๑ Única ₹ con régimen general cooperaciones reforzadas: previsión expresa en TFUE

\section{C.2. EL RÉGIMEN DE LAS COOPERACIONES REFORZADAS}

1) Régimen general

○ Art. 20.1, §1 TUE, p. 59

- Posibles sólo en el marco de competencias no exclusivas de la UE

- Límites materiales: Arts. 326-327 TFUE, p. 178

○ Art. 20.1, §2 TUE, pp. 59-60

- Finalidad cooperaciones reforzadas

- Abiertas permanentemente a todos los EEMM

o Confirmado en art. 328 TFUE, p. 178

2) Procedimiento:

- Nunca en ámbitos de competencia exclusiva de la UE

○ Art. 329.1 TFUE, p. 179: Procedimiento general (en todos los ámbitos competenciales restantes, salvo en la PESC)

○ Art. 329.2 TFUE, p. 179: Procedimiento especial en la PESC

3) Autorización de una cooperación reforzada: Art. 20.2 TUE, p. 60

○ Último recuso y mínimo de 9 EEMM 
○ Sistema de votación

- Art. 20.3 TUE, p. 60

- Art. 330 TFUE, p. 179 y mecanismo de flexibilidad en art. 333 TFUE, p. 180

- Art. 238.3 TFUE, p. 152

4. Eficacia cooperaciones reforzadas Art. 20.4 TUE, p. 60

○ Eficacia limitada ( $\approx$ eficacia relativa tratados internacionales)

$\odot$ Derecho de opting out para nuevos EEMM

- Históricamente, Suecia y el euro

5. Adhesión de más EEMM a una cooperación reforzada ya existente

○ Procedimiento general (salvo en la PESC) Art. 331.1 TFUE, p. 179

○ Procedimiento especial en la PESC Art. 331.2 TFUE, pp. 179-180

6. Primera cooperación reforzada existente: Reglamento (UE) no 1259/2010, de 20-XII-2010, por el que se establece una cooperación reforzada en el ámbito de la ley aplicable al divorcio y a la separación judicial (14 EEMM, incluida España)

7. Propuesta Comisión 28-XI-2011: Directiva sobre el impuesto a la transacciones financieras

○ Propuesta revisada el 19-II-2013: cooperación reforzada que vinculará a 11 EEMM (incluida España)

- $0.1 \%$ para acciones y bonos de los bancos

- $0.01 \%$ para derivados financieros de los bancos

- Se espera recaudar entre 30-35 billones $€$ por año 
1) Posibilidad de aplicar en la PESC cooperaciones reforzadas. Además:

2) Pese a regla de unanimidad en adopción de decisiones PESC, cláusula de "abstención constructiva" (el "No" de uno o varios EEMM no es un veto)

○ Art. 31.1 TUE, p. 64

3) En el núcleo duro de la Política Común de Seguridad y Defensa (PCSD)

○ Art. 42.5 TUE, p. 68 y Art. 44.1 TUE, p. 69

4) Novedad Tdo. Lisboa: Cooperación estructurada permanente en el ámbito de la PCSD

○ Arts. 42.6 y 46 TUE, pp. 68 y 70, respectivamente

(c) (1)(९) Esta obra está bajo una Licencia Creative Commons 